\title{
Context, intelligence and interactions for personalized systems
}

\author{
Liming Chen ${ }^{1}\left[\right.$ - Sarah Fallmann ${ }^{1} \cdot$ Diego López-de-Ipiña ${ }^{2} \cdot$ Chengsheng Pan $^{3} \cdot$ Huansheng Ning ${ }^{4}$
}

Published online: 31 August 2018

(c) Springer-Verlag GmbH Germany, part of Springer Nature 2018

\section{Introduction}

This special issue on Context, Intelligence and Interactions for Personalized Systems provides a snapshot of the latest research activities, results, and technologies and application developments focusing on the smart personalised systems in Ambient Intelligence and Humanized Computing. It is intended for researchers and practitioners from artificial intelligence (AI) with expertise in formal modeling, representation and inference on situations, activities and goals; researchers from ubiquitous computing and embedded systems with expertise in context-aware computing; and application developers or users with expertise and experience in user requirements, system implementation and evaluation. The special issue also serves to motivate application scenarios from various domains including smart homes and cities, localisation tracking, image analysis and environmental monitoring. For solution developers and providers of specific application domains, this special issue will provide an

Liming Chen

liming.chen@dmu.ac.uk

http://www.tech.dmu.ac.uk/ limingchen/

Sarah Fallmann

sarah.fallmann@dmu.ac.uk

Diego López-de-Ipiña

dipina@deusto.es

Chengsheng Pan

pancs@sohu.com

Huansheng Ning

ninghuansheng@ustb.edu.cn

1 School of Computer Science and Informatics, De Montfort University, Leicester, UK

2 Deusto Institute of Technology, University of Deusto, Bilbao, Spain

3 School of Information Engineering, Dalian University, Liaoning, China

4 School of Computer and Communication Engineering, University of Science and Technology Beijing, Beijing, China opportunity to convey needs and requirements, as well as obtain first-hand information on the latest technologies, prototypes, and application exemplars.

\section{Contributions of this issue}

This special issue consists of 12 high-quality research papers selected from a large number of submissions (126), each going through at least two rounds of strict peer reviews and significant consolidation. These papers represent the latest advances and development of research in the general context of Ambient Intelligence and Humanized Computing for smart human-machine systems.

The research topics covered in this special issue are wideranging, including context modelling and inference for smart environments (Alegre-Ibarra et al. 2018; Mulero et al. 2018; Nakahara and Beder 2018), localization tracking (Chen et al. 2018; Xin et al. 2018), optimization of energy consumption (Hammoud et al. 2018), behavior mining and activity recognition (Han et al. 2018; Zhang et al. 2018), image analysis (Wu et al. 2018), social event mining and mobile crowd sensing (Pan et al. 2018; Park 2018), and behavior-based privacy (Tao et al. 2018).

The application areas involved are diverse, including assistive living in smart environments (Alegre-Ibarra et al. 2018; Mulero et al. 2018; Nakahara and Beder 2018; Zhang et al. 2018), indoor and outdoor localization (Chen et al. 2018; Han et al. 2018; Xin et al. 2018), crowd-sourced big data analysis (Pan et al. 2018; Park 2018), privacy (Tao et al. 2018), energy usage optimization (Hammoud et al. 2018) and image segmentation (Wu et al. 2018). Details for each item are briefly described below.

While paper 'A Context-Aware and Self-Adaptive Offloading Decision Support Model for Mobile Cloud Computing System' (Nakahara and Beder 2018) by Flávio Akira Nakahara and Delano Medeiros Beder presents a contextaware and self-adaptive offloading decision support model based on application's time execution and energy consumption for decision-taking estimation to improve system 
execution; paper 'Towards Ambient Assisted Cities Using Linked Data and Data Analysis' (Mulero et al. 2018) authored by Rubén Mulero et al. investigates two methods to help Ambient Assisted Cities to deal with Mild Cognitive Impairments and Frailty. These methods discuss a citywide context manager and an individual care monitoring dashboard. In the paper 'Perspectives on engineering more usable context-aware systems' (Alegre-Ibarra et al. 2018) authored by Unai Alegre-Ibarra et al., the state-of-the-art conceptualisation of context was reviewed and a combination of revised and new definitions was introduced.

While paper 'FreeSense: Human-Behavior Understanding using Wi-Fi Signals' (Xin et al. 2018) authored by Tong Xin et al., presents a method for human indoor identification using Wi-Fi CSI signals. Influence patterns for human detection are captured by combining Principal Component Analysis, Discrete Wavelet Transform and Dynamic Time Warping techniques; paper 'A Three-stage Online Map-Matching Algorithm by Fully Using Vehicle Heading Direction' (Chen et al. 2018) by Chao Chen et al. propose a three stage online map-matching algorithm called SD-Matching for trajectory data matching. SD-matching takes the vehicle heading direction in consideration in every stage.

The paper 'Adaptive Power Switching Technique For Ultrasonic Motion Sensors' (Hammoud et al. 2018) by Abbass Hammoud et al. presents an adaptive power switching technique for ultrasound motion sensors for transmitter power level optimization to decrease energy consumption. Furthermore, an automatic sensing method can detect changes to trigger the process.

While in 'Driving Behavior Modeling and Evaluation For Bus enter and leave Stop Process' (Han et al. 2018) authored by Qingwen Han et al., special driving areas are distinguished by critical zone and a driving behavior model is constructed by using the bus enters and leave bus stop process. The approach includes driving behaviour establishment, good driving behavior discovery and current driving behavior evaluation; in paper 'Students Performance Modeling Based on Behavior Pattern' (Zhang et al. 2018) authored by Xi Zhang et al. a system modelling students' performance was established by using smart card readings to detect behaviour patterns and further extract statistical and relevance features for the performance modelling.

In the paper 'Grading Glioma by Radiomics with Feature Selection Based on Mutual Information' (Wu et al. 2018) authored by Yaping $\mathrm{Wu}$ et al., a prediction framework is proposed for grading of glioma based on radiomics. The system integrates a semi-automatic segmentation method, a de-redundancy algorithm, an elastic net feature selection and a linear prediction model.

While paper 'A Resource Recommender System based on User History and the Psychological Model' (Park 2018) authored by Jonghyun Park, investigates a customized resource recommendation system for mobile device users, reducing reasoning time and increase user satisfaction by a DISC physiological model; paper 'A Survey of RDF Management Technologies and Bechmark Datasets' (Pan et al. 2018) by Zhengyu Pan et al. discusses the state-of-theart Resource Description Framework storage and query technologies, and introduced and compared benchmark datasets.

The paper 'Gait based biometric personal authentication by using MEMS inertial sensors' (Tao et al. 2018) by Shuai Tao et al. presents a personal authentication method for MEMS inertial sensors in shoes for gait analysis. To distinguish different users a probabilistic neural network is used.

Acknowledgements This special issue was only possible by the help from various collaborators, which we would like to thank. Our gratitude goes to the Editor in Chief of the Journal of Ambient Intelligence and Humanized Computing, Prof. Vincenzo Loia, for his advice and support during the whole process of publication. Furthermore, we would like to thank the reviewers which took time to thoroughly review the papers and provide valuable feedback. We are very grateful for the contributions of all authors, without whom the presented special issue would not have been possible.

\section{References}

Alegre-Ibarra U, Augusto JC, Evans C (2018) Perspectives on engineering more usable context-aware systems. J Ambient Intell Humanized Comput. https://doi.org/10.1007/s1265 2-018-0863-7

Chen C, Ding Y, Xie X, Zhang S (2018) A three-stage online mapmatching algorithm by fully using vehicle heading direction. $\mathrm{J}$ Ambient Intell Human Comput. https://doi.org/10.1007/s1265 2-018-0760-0

Hammoud A, Deriaz M, Konstantas D (2018) Adaptive power switching technique for ultrasonic motion sensors. J Ambient Intell Human Comput. https://doi.org/10.1007/s 1265 2-018-0888-y

Han Q, Zeng L, Hu Y, Ye L, Tang Y, Lei J, Zhang X (2018) Driving behavior modeling and evaluation for bus enter and leave stop process. J Ambient Intell Human Comput. https://doi. org/10.1007/s12652-018-0802-7

Mulero R, Urosevi V, Almeida A, Tatsiopoulos C (2018) Ambient assisted cities using linked data and data analysis. J Ambient Intell Human Comput. https://doi.org/10.1007/s1265 2-018-0916-y

Nakahara FA, Beder DM (2018) A context-aware and self-adaptive offloading decision support model for mobile cloud computing system. J Ambient Intell Human Comput. https://doi. org/10.1007/s12652-018-0790-7

Pan Z, Zhu T, Liu H, Ning, H (2018) A survey of RDF managment technologies and bechmark datasets. J Ambient Intell Human Comput. https://doi.org/10.1007/s12652-018-0876-2

Park J-H (2018) A resource recommender system based on user history and the psychological model. J Ambient Intell Humaniz Comput. https://doi.org/10.1007/s12652-018-0807-2

Tao S, Zhang X, Cai H, Lv Z, Hu C, Xie H (2018) Gait based biometric personal authentication by using MEMS inertial sensors. 
J Ambient Intell Human Comput. https://doi.org/10.1007/s1265 2-018-0880-6

Wu Y, Liu B, Wu W, Lin Y, Yang C, Wang M (2018) Grading glioma by radiomics with feature selection based on mutual information. J Ambient Intell Human Comput. https://doi.org/10.1007/ s12652-018-0883-3
Xin T, Guo B, Wang Z, Wang P, Yu Z (2018) FreeSense : humanbehavior understanding using Wi-Fi signals. J Ambient Intell Human Comput. https://doi.org/10.1007/s12652-018-0793-4

Zhang X, Sun G, Pan Y, Sun H, He Y, Tan J (2018) Students Performance Modeling Based on Behavior Pattern. J Ambient Intell Human Comput. https://doi.org/10.1007/s12652-018-0864-6 